\title{
Two-state versus continuous-state stimulus representations: A test based on attentional constraints
}

\author{
MARILYN L. SHAW \\ Bell Laboratories, Murray Hill, New Jersey \\ and Rutgers-The State University, New Brunswick, New Jersey \\ ROBERT M. MULLIGAN \\ Rutgers-The State University, New Brunswick, New Jersey \\ and \\ LAWRENCE D. STONE \\ Daniel H. Wagner Associates, Paoli, Pennsylvania
}

\begin{abstract}
New predictions of a class of two-state and a class of continuous-state attention models are described and then tested in an experiment requiring subjects to detect and locate a target occurring in one of four possible locations. The model predictions are tested by comparing the effect of focused- versus divided-attention instructions on the probability of correctly locating a target. The class of two-state models predicts that target localization performance will be better in the focused- than in the divided-attention condition, while the class of continuousstate models predicts that performance in the divided-attention condition will equal or exceed that in the focused attention condition. Results from an experiment testing these predictions favor the continuous-state models.
\end{abstract}

In this paper we present an analysis of two alternative classes of attention models. Both classes of models assume that, at any given instant, the human observer has a limited amount of processing resources, capacity, or attention. The models differ, however, in their assumptions concerning the internal-strength representation of the stimulus used in deciding whether a target is present in a visual search task. In the class of two-state models, it is assumed that the internal-strength representation is categorical: target stimulus present or absent. Since only two gradations of strength are allowed, this categorical stimulus code is modeled as a two-valued random variable. This formulation does not, of course, exclude the possibility that the internal-strength representation of the same stimulus is better treated as a more than two-valued random variable at other points in time or other stages of processing. However, this class of models, like early threshold models, does exclude the possibility that judgmental standards can alter the

This research was sponsored by National Science Foundation Grants BNS 77-26296 and BNS 79-24885 to Rutgers University. The authors gratefully acknowledge the many helpful comments made by D. H. Krantz. Requests for reprints should be addressed to the first author at Bell Laboratories, 600 Mountain Avenue, Murray Hill, New Jersey 07974. probability distribution of the two-state variable. In the class of continuous-state attention models, the internal strength representation used in decision making is modeled as a continuous-valued random variable. This treatment is based on the notion of a stimulus code in which a continuous gradation of sensory strength is allowed and the observer has the option of choosing a judgmental criterion anywhere along this continuum.

Our interest in these classes of models was prompted by work on attending to multiple sources of information (e.g., Eriksen \& Spencer, 1969; Estes \& Taylor, 1964; Gardner, 1973; Kinchla, 1969, 1974; Mulligan \& Shaw, 1980, 1981; Shaw, 1980, 1982, 1983; Shiffrin \& Gardner, 1972). The analysis presented herein was inspired chiefly by work in formal search theory (Kadane, 1971; Koopman, 1957; Mela, 1961; Stone, 1975; Tognetti, 1968) concerned with performance optimization in the detection and location of targets. We were guided also by analyses in psychophysics of two-state and continuous-state detection models (Green \& Swets, 1966; Krantz, 1969; Luce, 1963, 1960; Wickelgren, 1967). The outcome of our analysis is the derivation of testable predictions which discriminate between these two classes of attention models. We will begin by describing (1) the relation of our work to earlier work in psychophysics, (2) the attention research 
that led us to the problem, and (3) the work in search theory that pointed to a possible solution. This brief review will be followed by a description of the experimental paradigm, and finally presentation of the formal models and their predictions.

\section{Research in Psychophysics}

The issue of whether internal strength representations are two-state or continuous-state arose when signal detection theory was introduced into psychology. Krantz (1969) has reviewed the numerous proposals for tests to discriminate between these two classes of models. Probably the best of these proposals relies on predictions for experiments in which subjects make both a detection and recognition response. The critical prediction for twostate models is that recognition performance on nondetection trials should be at the chance level. In contrast, continuous-state models predict that recognition performance can be above chance on signal trials on which the subject responds "no." Unfortunately, quantitative comparisons of these models and their predictions brought about no sharp distinction between them (Rollman \& Nachmias, 1972). In this paper, we show how data from a simultaneous detection and localization experiment can lead to a sharp distinction between two- and continuous-state models. The predictions we derive are based on two assumptions that processing capacity is limited and dividing attention among multiple sources decreases the internal signal-to-noise ratio associated with each source.

\section{Research in Attention}

In a recent series of experiments, one of us (Shaw, 1983) examined the issue of whether dividing attention among different spatial locations causes losses of information. Two specific attention models postulating losses of information were tested: the capacity-allocation model and the sample-size model. For the present discussion, the most important difference between these two is that the first is a twostate model and the second is a continuous-state model. The capacity-allocation model is based on the assumption that the conditional probability of detection of a target is the cumulative exponential function. The sample-size model is based on the assumption that each internal random variable has a Gaussian probability function. In these models, the loss of information caused by dividing attention is reflected by a change in the parameters of the underlying probability distributions. Two other specific models in which the division of attention does not cause losses of information were also considered. Both these models assume that the internal random variables are continuous; one is based on the Gaussian probability function and the other on the exponential. These four models were compared to a "boundary" function which gives the

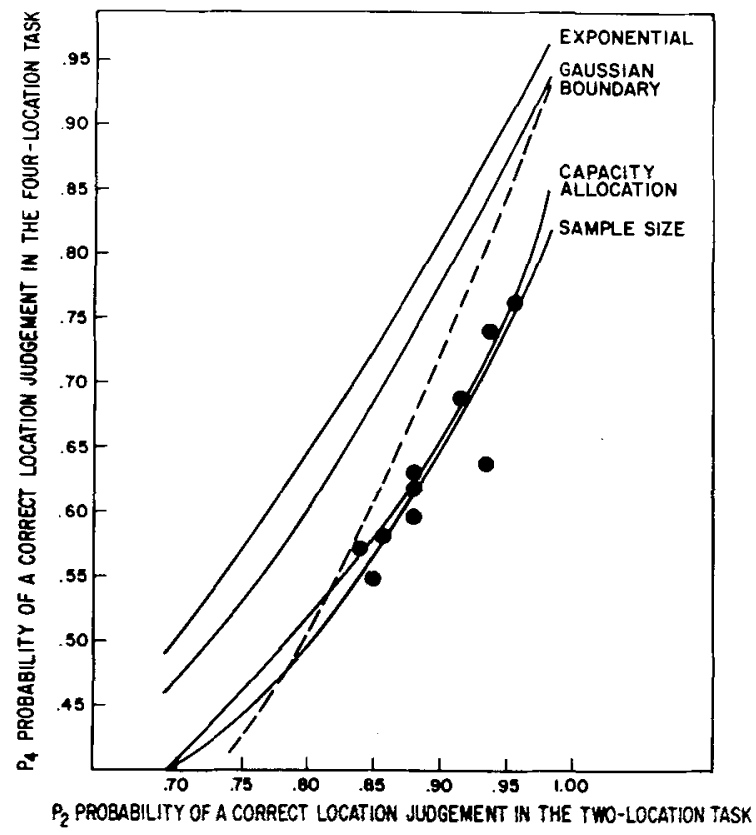

Figure 1. The relation between $P_{2}$ (probability of a correct location judgment in the two-location task) and $P_{4}$ (same probability in the four-location task) predicted by several models and data obtained by Shaw (1983) in a visual search experiment.

greatest possible change in performance that can occur as a function of display size, if division of attention does not cause losses of information. More detail on derivation of the boundary function and the alternative models is given in Shaw (1980).

Predictions of the four alternative models and the boundary function are presented in Figure 1 together with data from several experiments reported in Shaw (1983). In the figure, theoretical probability of a correct location response for two possible stimulus locations is plotted against this probability for the case of four stimulus locations for each of the models. Data from individual subjects are indicated by the dots. The data clearly favor the sample-size and capacity-allocation models, but it is also evident that data from this type of experiment cannot distinguish between them. Since both models give a good account of the data, yet differ considerably in their underlying assumptions, it is of interest to discriminate between them.

\section{Research in Search Theory}

In Appendix 1 of this paper, predictions are derived which distinguish between generalized versions of the sample-size and capacity-allocation models. The ideas underlying the derivation of these predictions grew out of an examination of the treatment of target detection and localization problems in formal search theory (Kadane, 1971; Koopman, 1957; Stone, 1975). In particular, our analysis draws upon the distinction between two possible search 
objectives: detection and whereabouts. In a detection search, the objective is to maximize the probability of correctly deciding whether a target is present or absent in at least one location, regardless of where it is. In a whereabouts search, the objective is to maximize the probability of correctly deciding which location, if any, contains the target. The formal models from search theory that we draw upon assume that a target is (1) present in one of the $n$ locations, (2) either detected or not detected, and (3) never detected in a location when it is not there (no false alarms). It is further assumed that there is a limited resource (attention, processing capacity, search effort, search time) available for processing, and that the probability of detection in a given location depends upon the amount of resource allocated there (the greater the resource, the greater the detection probability). Each type of search (detection and whereabouts) can be viewed as an optimization problem in which the observer must decide how to allocate the limited resource among locations in order to maximize the appropriate response probability.

To illustrate these two optimization problems, let us take an example. Suppose there is a gold coin lost in one of $\mathbf{n}$ boxes, and the prior probability that the coin is in each box is known. We are permitted a total of $\mathbf{K}$ draws, and each draw is made with replacement. After each draw, the probabilities for all boxes are revised; that is, a set of posterior probabilities is computed. For detection search, we wish to find an allocation of these $K$ draws among the boxes in order to maximize the probability of drawing the gold coin. It can be shown (cf. Stone, 1975) that the optimal allocation of draws for detection search places the next draw in the box with the greatest posterior probability, that is, the box that is most likely to contain the coin, given failure to find it so far.

For whereabouts search, rather than trying to maximize the probability of drawing the gold coin within $\mathrm{K}$ draws, we wish to maximize the probability of correctly specifying the box containing the target. There are two possible paths to a successful whereabouts search. First, if the coin is detected on one of the $\mathrm{K}$ draws, it will be correctly localized. Second, if after $\mathbf{K}$ draws the coin is not found, we are allowed to guess which box contains the coin. How should the $\mathrm{K}$ draws be distributed in this case? As in detection search, we must first identify the box that is most likely to contain the coin. Unlike detection search, however, in whereabouts search, no draws are allocated to this box. All of the resource ( $\mathrm{K}$ draws) is allocated among the remaining $\mathrm{n}-1$ boxes so as to maximize the probability of drawing the coin, if it is in one of these remaining boxes. If the prior probabilities for the boxes are equal, then one box is arbitrarily chosen to be ignored and the $\mathrm{K}$ draws are divided equally among the remain- ing boxes. If after $K$ draws the coin is not located in one of the $n-1$ boxes, then the ignored box is guessed. The reason this allocation of draws is optimal is as follows: If the coin is in the ignored box, then it will be correctly located by the guess; so nothing is gained by allocating draws to it. If it is not in the ignored box, then, of course, one does not wish to allocate draws there. In either case, draws allocated to the ignored box are effectively wasted.

\section{Summary of New Results}

Work in search theory on the optimization of detection and whereabouts search has concentrated on the case of discrete effort (resource) applied to discrete locations. The "gold coin" example is an illustration of this case in which individual draws are the discrete units of effort. While some models of detection search have considered the case of continuous effort and/or continuous probability density functions, whereabouts models have always treated these parameters as discrete-valued. In order to adapt this theoretical framework to models of attention, in which attention is usually regarded as a continuous rather than a discrete resource, we have derived detection and whereabouts predictions for the case of continuous effort applied to discrete locations. One set of predictions is based on the notion, common to the search-theory models, that the internal representation of information at each location is discrete (two-state). In addition, a completely new set of predictions is provided for a class of models assuming continuous-valued internal representations. It should also be pointed out that the probability function for the class of two-state attention models to be considered allows both false alarms and misses. This is a departure from the usual assumption in search theory that there are no false alarms. For the continuous-state model, we assume that the probability function of an internal representation is unimodal and symmetric. The principal characteristics of these two classes of attention models and search-theory models are summarized in Table 1.

It is shown in Appendix 1 that, for the case of continuous effort (attention, processing capacity, etc.), the problem of whether both detection and whereabouts searches can be optimized by the same allocation of resources depends upon whether the representation of strength acquired during processing or search is continuous- or two-valued. If the strength measure can be represented as a twovalued random variable (two-state model), then under certain circumstances detailed in Appendix 1, each optimization problem requires a different allocation of resources. If, however, the strength is represented as a continuous-valued random variable (continuous-state models), then simultaneous optimization of detection and whereabouts is pos- 
Table 1

Comparison of Two-State and Continuous-State Attention Models

\begin{tabular}{|c|c|c|c|}
\hline & Two-State Model & $\begin{array}{c}\text { Continuous-State } \\
\text { Model }\end{array}$ & Search Theory \\
\hline $\begin{array}{l}\text { Internal strength } \\
\text { representation }\end{array}$ & $\begin{array}{l}\text { Two-valued } \\
\text { (detect, nondetect) }\end{array}$ & Continuous-valued & $\begin{array}{l}\text { Two-valued } \\
\text { (detect, nondetect) }\end{array}$ \\
\hline Attention & Continuous-valued & Continuous-valued & Integer-valued \\
\hline $\begin{array}{l}\text { Probability function } \\
\text { of the internal } \\
\text { representation }\end{array}$ & $\begin{array}{l}\mathrm{P}(\text { detect } \mid \text { target absent })>0 \\
\mathrm{P}(\text { nondetect } \mid \text { target present })>0\end{array}$ & $\begin{array}{l}\text { Unimodal and } \\
\text { symmetric }\end{array}$ & $\begin{array}{l}P(\text { detect } / \text { target } \\
\text { absent })=0 \\
P(\text { detect/target } \\
\text { present })>0\end{array}$ \\
\hline $\begin{array}{l}\text { Optimal allocation } \\
\text { for whereabouts } \\
\text { vs. detection search }\end{array}$ & $\begin{array}{l}\text { Different allocation } \\
\text { required by these } \\
\text { two objectives }\end{array}$ & $\begin{array}{l}\text { Same allocation } \\
\text { optimizes these } \\
\text { two objectives }\end{array}$ & $\begin{array}{l}\text { Different allocation } \\
\text { required by these } \\
\text { two objectives }\end{array}$ \\
\hline
\end{tabular}

* Parameters of the distribution function depend on whether the target is present or absent and on how much attention is allocated to a given location.

sible. In the continuous-state model, sampling always gives continuous gradations of information instead of the binary information provided in a twostate model. It is this difference between the models that leads to the differences in optimal strategy.

We have also considered models between two-' state and continuous-state models. In Shaw (1982), predictions from a three-state model were tested against predictions for detection performance derived from two-state and continuous-state models. The three-state model was rejected by data from a letter-detection task similar to the one reported here. Our explorations of models between three-state and continuous-state models did not result in any differential predictions for detection and whereabouts searches.

\section{EXPERIMENTAL PARADIGM}

Before describing the formal models to be tested, let us introduce some notation and briefly outline the experimental paradigm. The internal-strength representation of the stimulus at each location will be regarded as a random variable. Each random variable, $X_{k i}$, is one of two types-target $(i=1)$ or nontarget $(i=0)$-depending on whether a target or nontarget is present at location $L_{k}$. The $X_{k i} s$ may be dependent or independent. The pattern of types of random variables over $\mathbf{n}$ locations is denoted by $S_{i_{1} i_{2} \ldots i_{n}}$, where $i$ again assumes values of 0 or 1 for each $L_{k}$.

In the experiment, the subject's task is to search an array of letters for a target letter. Arrays contain either four letters positioned at the corners of an imaginary square or two letters at opposite ends of the diagonals of the square. There are four possible $S_{i j}$ patterns for each of the two-letter displays. For both positively and negatively sloped diagonals, the first subscript, $i$, refers to the upper end of a diagonal (i.e., top left or top right of the square), and the second subscript, $j$, refers to the lower diag- onal position. There are 16 possible four-location displays, denoted by $S_{i j k \ell}$, where $i$ and $j$ denote the upper and lower positions of the positive diagonal and $k$ and $l$ the upper and lower positions of the negative diagonal.

On each trial, regardless of display size, subjects are required to make two responses-a "yes-no" detection response and a target localization (whereabouts) response. When displays contain two letters, the subject chooses an upper or lower diagonal position for the whereabouts response. But, when the display contains four letters, the subjects choose either the positive or negative diagonal. Limiting response alternatives to the two diagonals rather than the four specific locations was forced by hardware limitations. Subjects are required to make both a detection response and a whereabouts response on every trial, even when the detection response is "no." There are two kinds of attention instructions for the four-location displays. With focused-attention instructions, the subject is told to attend to just one diagonal and ignore the other diagonal. For the detection response, the subject is instructed to respond "yes" or "no" based only on information in the attended diagonal. For the whereabouts response, however, the subject is told to guess the unattended diagonal if the target is not detected in the attended diagonal. With dividedattention instructions, the subject is told to attend equally to all four locations. For both detection and whereabouts responses, the subject is instructed to use information from all locations in making a choice.

\section{THE FORMAL MODELS}

Model assumptions can be divided into two groups: representation assumptions and decisionmaking assumptions. The former are concerned with the stimulus-strength measure (two-state vs. continuous) used in decision making and how at- 
tention affects this code. The decision-making assumptions are concerned with how a response is selected given the pattern of strength measures.

Specific decision models for the detection response have been worked out, and are presented in Appendix 2. We do not elaborate on them here, however, since it is not necessary to assume a specific decision model for the detection response in order to derive predictions which will discriminate between two-state and continuous-state attention models. For purposes of the models we will test, the detection data will be used simply to determine if subjects are following instructions to ignore one diagonal in the focused-attention condition. In previous papers (Mulligan \& Shaw, 1980; Shaw, 1980, 1982), we have considered several alternative decision models for detection with multiple sources of information. These models, and an analysis of detection data from the present experiment, are presented in Appendix 2.

\section{A Class of Two-State Models}

\section{Representation Assumptions}

First, attention is assumed to be a finite quantity $\Phi$. We denote by $\phi_{k}$ the amount of attention allocated to $L_{k}$. It is assumed that $\Phi$ may be partitioned among locations, and this does not change the total amount:

$$
\Phi=\sum_{k=1}^{n} \phi_{k}
$$

This assumption has been supported in tests of the capacity-allocation model (Shaw \& Shaw, 1977) and the sample-size model (Shaw, 1983).

The third representation assumption concerns the probability of a detection state for location $L_{k}$, given that the target is there, $X_{k 1}=1$, and $\phi_{k}$ has been allocated there: $b\left(\phi_{k}\right)$. It is assumed that $b\left(\phi_{k}\right)$ is continuous, concave, and increasing with $\phi_{k}$. Naturally, $1-b\left(\phi_{k}\right)$ is the probability of missing the target in location $\mathrm{L}_{\mathbf{x}}$. The capacity-allocation model makes the specific assumption that $b\left(\phi_{k}\right)=1-e^{-\phi_{k}}$.

The fourth assumption concerns the probability of a nondetection state for location $L_{k}$, given that a nontarget is there, $X_{k 0}=0$, and that $\phi_{k}$ has been allocated there, $q\left(\phi_{k}\right)$. In the capacity-allocation model, it is assumed that $\mathrm{q}\left(\phi_{k}\right)=1$; that is, false alarms are assumed not to occur. It will be assumed here, however, that $q\left(\phi_{k}\right)$ is continuous, increasing, and nonzero. The probability of a false alarm in $L_{k},\left(X_{k 0}=1\right)$, is written as $1-q\left(\phi_{k}\right)$.

\section{Decision-Making Assumptions}

Decision models for the whereabouts task are outlined below for each of three experimental conditions: two-location displays, four-location dis- plays with focused attention, and four-location displays with divided attention. These decision models assume that the target is equally likely to appear in each location or diagonal. With twolocation displays, if the stimulus produces a detect state in one location, then that location is selected for the whereabouts response. If both locations or neither location results in a detect state, then one of the locations is chosen at random.

With four-location displays, decision-making assumptions differ for focused-attention (FA) versus divided-attention (DA) instructions. In the FA condition, if either location in the attended diagonal results in a detect state, then the attended diagonal is chosen for the whereabouts response; otherwise, the nonattended diagonal is selected. With DA instructions, the subject is assumed to choose the diagonal with the greatest number of detect states, or to pick randomly when the diagonals have the same number of detect states.

\section{Two-State Model Predictions}

Whereabouts response. The two-state attention model predicts that the probability of a correct whereabouts response will be greater in the FA than in the DA condition. This is analogous to the theorem we described from search theory in which whereabouts performance is optimized when one of the locations (boxes) is ignored. As we have already noted, however, some assumptions of the attention models differ from those made in formal search theory (see Table 1 for these differences). In Appendix 1, it is formally demonstrated that, for the case of two locations and a uniform prior probability distribution on target location, the twostate model predicts that, on the average, the probability of a correct whereabouts response will be greater for the focused-attention condition than for the divided-attention condition. Thus, if we let $\operatorname{Pr}(\mathrm{CW} \mid \mathrm{FA})$ and $\operatorname{Pr}(\mathrm{CW} \mid \mathrm{DA})$ denote the probability of a correct whereabouts response in the FA and DA conditions, respectively, the twostate model predicts that:

$$
\operatorname{Pr}(C W \mid \text { FA })>\operatorname{Pr}(C W \mid D A) \text {. }
$$

If one does not assume a uniform prior probability function, then the question of whether focused or divided attention is the superior strategy depends in a somewhat complicated way upon the relation between $b(\phi)$ and $q(\phi)$. (See Appendix 1).

The inequality in Equation 2 is valid for fourletter displays with a single target or two targets on the same diagonal. If a target appears in both diagonals, then the subject is correct no matter what the choice; conversely, if no targets are present, then no choice of locations can be correct. Here, we analyze data only from trials in which a single target appears. 
Whether the prediction in the above inequality can be observed depends on the size of the difference between these two probabilities and the precision of the data collected. We computed the expected difference in $\operatorname{Pr}(\mathrm{CW} \mid \mathrm{FA})$ and $\operatorname{Pr}(\mathrm{CW} \mid \mathrm{DA})$ for the capacity-allocation model as a function of the difference between the false-alarm rate when attention is focused versus when attention is divided. ${ }^{1}$ This difference $[\operatorname{Pr}(\mathrm{CW} \mid \mathrm{FA})-\operatorname{Pr}(\mathrm{CW} \mid \mathrm{DA})]$ is plotted in Figure 2 for two different values of $\Phi$, the total capacity. The predicted difference ranges from .05 to .35 . Thus, it appears that one can ensure that the expected difference for this model can be made sufficiently large by adjusting display duration such that the estimated $\Phi$ corresponds to predictions of $\operatorname{Pr}(\mathrm{CW} \mid \mathrm{FA})-\operatorname{Pr}(\mathrm{CW} \mid \mathrm{DA})>.10$, if the two-state model holds. In our experiments, estimates of $\Phi$ ranged between 2.3 and 3.5, depending on the subject.

Detection response. The relationship expressed in Equation 2 depends completely on whether subjects can and do follow the instructions to attend to a single diagonal in the focused-attention conditions. To check this, we compare detection performance in the focused-attention condition to that in the two-location condition as indicated in the three equations below. If subjects are following instructions, then in each of these conditions they need process only two locations. If other differences between the conditions exert little or no influence on detection performance, then detection performance for the four-location stimuli having no targets in the attended diagonal should be identical to that in the $S_{\infty}$ condition. This should hold whether targets are present in (Equation 3a) or absent from (Equation $3 b$ ) the ignored diagonal. In addition, detection performance for a diagonal with one target should be identical in the twolocation and four-location conditions (Equation 3c). These three tests for focused attention are stated below in terms of the probability of a "no" response:

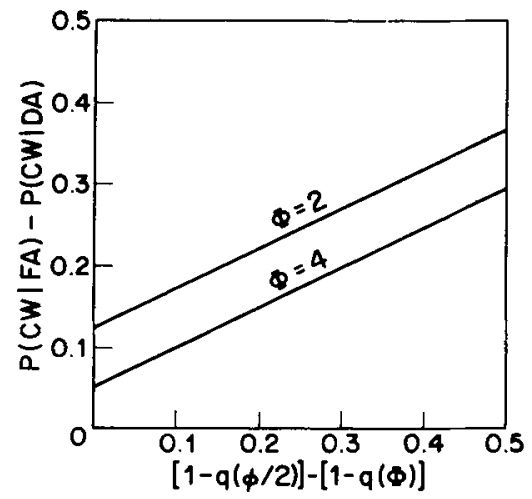

Figure 2. Predictions of the capacity-allocation model: the difference between $\operatorname{Pr}(\mathrm{CW} \mid \mathrm{FA})$ and $\operatorname{Pr}(\mathrm{CW} \mid \mathrm{DA})$ as a functon of the difference in the false-alarm rate for focused versus divided attention for two different values of total capacity, $\Phi$.

$$
\begin{aligned}
& \operatorname{Pr}\left(\text { no } \mid S_{00}\right) \\
& =\operatorname{Pr}(\text { no } \mid F A ; \text { distractors in attended } \\
& \text { diagonal and one target in ignored } \\
& \text { diagonal) } \\
& \operatorname{Pr}\left(\text { no } \mid S_{\infty 0}\right) \\
& =\operatorname{Pr}\left(\text { no } \mid F A ; S_{0000}\right) \\
& \operatorname{Pr}\left(\text { no } \mid \mathbf{S}_{01}, \mathbf{S}_{10}\right) \\
& =\operatorname{Pr} \text { (no } \mid \text { FA; one target in attended } \\
& \text { diagonal and distractors in ignored } \\
& \text { diagonal) }
\end{aligned}
$$

The data are expressed in terms of the probability of a "no" response, rather than probability of a "yes" or probability correct, in order to facilitate the decision-model analysis of the detection data presented in Appendix 2. It is easily demonstrated that these same tests can be expressed in terms of any of these response measures.

\section{A Class of Continuous-State Attention Models}

\section{Representation Assumptions}

Again, attention is assumed to be a finite quantity, $\Phi$, and it is assumed to conform to Equation 1. Let $f_{t}(X)$ and $f_{d}(X)$ denote the probability density functions of the random variables $X_{\mathrm{k}_{1}}$ and $\mathrm{X}_{\mathrm{t} 0}$ associated with $L_{k}$ when a target or a distractor is present, respectively. It is assumed that $f_{t}$ and $f_{d}$ are symmetric, unimodal distributions, and have the same functional form. For convenience, we assume that the mean of the target random variable is greater than the mean of the distractor random variable. Attention is assumed to influence the variability (noise level) of the internal strength representation. Specifically, we assume that $\tilde{o}_{k}^{2}$, the variance of the internal strength representation at location $L_{x}$, is inversely proportional to the amount of attention allocated there. Thus,

$$
\tilde{\sigma}_{k}^{2}=\frac{\sigma_{k}^{2}}{\Phi_{k}}
$$

where $\sigma_{\mathrm{k}}^{2}$ is a constant that depends upon the stimulus conditions. ${ }^{2}$ Evidence that the assumption expressed by Equation 4 is consistent with data from set-size studies has been provided by the fact that the data points in Figure 1 do indeed fall on or near the function predicted by the sample-size model. This is because the curve for the sample-size model in Figure 1 was derived from the assumption that $\Phi$ is a constant and that $\Sigma \phi_{x}=\Phi$, as well as the assumption that internal strength values are Gaussian. 
An attention model very similar to this model has been studied by Luce and Green (see Green \& Luce, 1973; Luce, 1977) in auditory detection and magnitude estimation paradigms.

\section{Decision-Making Assumptions}

Most continuous-state models assume that when forced to choose between $n$ random variables, the subject chooses the response whose associated random variable is the maximum (or minimum) of the $n$. We likewise make this assumption; thus, the probability that location $L_{k}$ is chosen, given $S_{i j}$, is given by

$$
\operatorname{Pr}\left(\text { “ } L_{k} \text { "' } \mid S_{i j}, X_{k}\right)=\operatorname{Pr}\left[X_{k}=\max \left(X_{1 i}, X_{2 j}\right)\right] \text {. }
$$

With four-letter displays and focused-attention instructions, the subject is assumed to choose the attended diagonal whenever one of the associated random variables exceeds its criterion; otherwise, the subject chooses the unattended diagonal.

In the divided-attention condition, we let $r=$ $\max \left(X_{1 i}, X_{2 j}\right)$ denote the maximum of the random variable associated with the positive diagonal, and $s=\max \left(X_{3 k}, X_{41}\right)$ denote the same quantity for the negative diagonal. It is assumed that the subject chooses the positive diagonal if $r=\max (r, s)$ and the negative diagonal if $s=\max (r, s)$.

\section{Continuous-State Model Predictions}

Whereabouts response. In contrast with the twostate model, the continuous-state models make the prediction that the same allocation of attention optimizes both whereabouts probability correct and detection probability correct (see Appendix 1). This predicts that subjects' whereabouts performance in the focused-attention conditions (ignoring a location to be guessed) should be equal to or poorer than their performance when they are in the divided-attention condition. Thus, the continuousstate model predicts that

$$
\operatorname{Pr}(C W \mid D A) \geqslant \operatorname{Pr}(C W \mid F A) .
$$

Detection response. Again, the validity of the inequality (6) depends completely on whether subjects can and do follow the instructions to attend to a single diagonal in the focused-attention conditions. As in the case of the two-state model, if subjects are following instructions, detection performance in the focused-attention condition should equal that in the two-letter condition. The continuous-state model and its predictions are presented in greater detail in Appendix 1 .

\section{Summary of Predictions}

The two classes of attention models make contrasting predictions for the probability of a correct where- abouts response for the different attention instructions we use. The two-state models predict that the whereabouts response will be superior in the focusedattention condition, whereas the continuous-state models predict that performance in the focused attention condition will either be the same as or inferior to performance in the divided attention condition. Data from the detection response is used simply to confirm the assumption that subjects do indeed ignore the unattended diagonal when so instructed.

\section{EXPERIMENT}

\section{Method}

\section{Subjects}

Four adults, three females and one male, each with normal or corrected-to-normal vision, served as observers. They were paid $\$ 3 / \mathrm{h}$.

\section{Stimuli}

The stimulus displays were similar to those used previously by Shaw (1983). Both two-location and four-location displays were used. The stimulus at each location consisted of a single letter flanked by dollar signs. In the two-location display, the threecharacter strings appeared at the ends of an imaginary diagonal, separated by $1.25 \mathrm{deg}$ of visual angle. In half of these displays, the characters appeared at the opposite ends of the positive diagonal (at the lower-left and upper-right of the screen); in the other half, they appeared at opposite ends of the negative diagonal (upper left, lower right). Four-location displays contained character strings at all four positions described by the ends of the two intersecting diagonals.

Varied mapping (Schneider \& Shiffrin, 1977) was employed; that is, the target letter changed from trial to trial. Both targets and nontargets were selected randomly on each trial from the following group: $\mathrm{F}, \mathrm{H}, \mathrm{J}, \mathrm{K}, \mathrm{L}, \mathrm{M}, \mathrm{N}, \mathrm{T}, \mathrm{V}, \mathrm{W}, \mathrm{X}, \mathrm{Y}$, and $\mathrm{Z}$. Each letter was used equally often as target and nontarget, and each appeared equally of ten in each position.

The a priori stimulus probability distribution for the two-letter display conditions was as follows: $\operatorname{Pr}\left(\mathrm{S}_{00}\right)=.40, \operatorname{Pr}\left(\mathrm{S}_{01}\right)=.25$, $\operatorname{Pr}\left(\mathrm{S}_{10}\right)=.25$, and $\operatorname{Pr}\left(\mathrm{S}_{11}\right)=.10$.

In the four-letter conditions, the stimulus probabilities chosen to approximate independence were as follows: $\operatorname{Pr}\left(\mathrm{S}_{0000}\right)=.40$, $\operatorname{Pr}\left(\mathrm{S}_{0001}\right)=.13, \operatorname{Pr}\left(\mathrm{S}_{0010}\right)=.13, \operatorname{Pr}\left(\mathrm{S}_{0100}\right)=.13, \operatorname{Pr}\left(\mathrm{S}_{1000}\right)=.13$, $\operatorname{Pr}\left(\mathrm{S}_{0101}\right)=.02, \operatorname{Pr}\left(\mathrm{S}_{0110}\right)=.02, \operatorname{Pr}\left(\mathrm{S}_{1001}\right)=.02$, and $\operatorname{Pr}\left(\mathrm{S}_{1010}\right)=.02$. Thus, the probability of a target on only the negative diagonal was .26 , on only the positive diagonal, .26 , and on both diagonals, .08. Note that two targets never appeared on the same diagonal and that stimulus patterns with three and four targets did not appear. (They occur with such small frequency in the independent case that we did not include them.)

\section{Procedure}

Subjects viewed the displays binocularly through a Tektronix viewing hood mounted $87 \mathrm{~cm}$ from the front surface of a Tektronix Model 610 cathode ray tube. The CRT was under the control of a DEC PDP-12 computer, using its standard letter format.

Each trial began with a 2-sec presentation of the letter chosen as target, followed by a 3-sec fixation period and then the 10-msec stimulus display. The fixation patterns consisted of two or four dots corresponding to the display locations, and an additional dot at the center of the display, that is, at the point of intersection of the two diagonals. The subjects were instructed to prepare for each display presentation by fixating the center dot. The importance of maintaining consistent fixation on all trials in all session types was stressed. Display presentation was followed by a $2-\mathrm{sec}$ period in which a "yes-no" detection response was made, a 2 -sec period in which a location judgment was made, and a 2 -sec feed- 
back period during which the target letter and stimulus pattern were redisplayed.

On two-location trials, the location judgment required a forced choice between the two stimulus locations. On four-location trials, the observer's task was to specify in which diagonal-positive or negative-the target had appeared. In both conditions, subjects were required to make a location judgment regardless of the outcome of their detection judgment.

Stimulus displays were presented in three types of sessions, corresponding to the three attentional strategies subjects were instructed to follow. In a divided-attention (DA) session, the subjects were instructed to divide their attention equally and simultaneously between all (either two or four) display locations, so as to maximize correct detection responses. In a focused-attention, positive-diagonal (FAP) session, the subjects were required to attend only to the positive diagonal and to ignore the other two locations. They were told to base their "yes-no" responses solely on the attended diagonal. For the localization response, they were instructed to choose the positive diagonal if the detection response was positive and to guess the negative diagonal otherwise. Similar instructions were given for the focused-attention, negative-diagonal (FAN) sessions, except that subjects were to base their responses on information from the negative diagonal.

Each DA session consisted of 20 unrecorded practice trials followed by three blocks of 152 trials-a positive-diagonal twolocation block, a negative-diagonal two-location block, and a four-location block. Order of blocks within sessions was counterbalanced. Subjects received 10-min breaks between blocks of trials.

Each FA session consisted of 50 unrecorded two-location trials, followed by two blocks of 152 four-location trials. The subjects followed the same instructions (FAP or FAN) for both blocks within a session. The two-location trials, always presented first, were given to help the subjects focus their attention on the appropriate diagonal for that session. The subjects reported this to be a useful procedure in helping them to follow whereabouts instructions. As an additional aid, the fixation pattern on four-location trials indicated only the diagonal to which the subject should attend.

Data were collected in 17 sessions ( 9 DA, 4 FAP, and 4 FAN), with FA and DA sessions alternated. In the 9 DA sessions, a total of 2,736 two-location trials (1,368 for each diagonal) and 1,368 four-location trials were presented. In the 8 focused-attention sessions, 1,216 FAP and 1,216 FAN trials were presented.

Prior to data collection, all subjects had participated in 10 practice sessions-6 DA, 2 FAP, and 2 FAN-a minimum of 608 trials per condition. During the first two sessions, both of which were DA sessions, display durations were gradually decreased from 100 to $10 \mathrm{msec}$. Thereafter, DA and FA sessions were alternated. During these practice sessions, display luminance was differentially adjusted for each subject to achieve the desired accuracy levels.

\section{Results}

Response probabilities (sample size in parentheses) are presented in Table 2 for all subjects and experimental conditions. Standard deviations for these estimated probabilities ranged from .03 to .009 , depending on the value of the estimate and the number of observations. Note that whereabouts performance is expressed in terms of probability correct, but detection performance, for reasons mentioned previously, is expressed in terms of the probability of a "no" response.
Table 2

Response Probabilities

\begin{tabular}{|c|c|c|c|c|c|c|c|c|}
\hline \multirow[b]{2}{*}{ SUBJECT } & \multicolumn{5}{|c|}{ Probability of a "No" Response } & \multicolumn{3}{|c|}{$\begin{array}{l}\text { Probability of a Correct } \\
\text { Whereabouts Response }\end{array}$} \\
\hline & $P_{\infty}$ & $P_{10}$ & $P_{01}$ & \multicolumn{2}{|c|}{$P_{11}$} & \multicolumn{2}{|c|}{ Upper } & Lower \\
\hline \multicolumn{9}{|c|}{ Two Locations } \\
\hline \multicolumn{9}{|c|}{ Positive Diagonal } \\
\hline 1 & $.90(392)$ & $.09(256)$ & $.12(252)$ & .01 & 106) & .960 & 56) & $.95(252)$ \\
\hline \multirow[t]{2}{*}{$\begin{array}{l}2 \\
3 \\
4\end{array}$} & $\begin{array}{l}.85(419) \\
.89(417) \\
.85(410)\end{array}$ & $\begin{array}{l}.16(264) \\
.11(266) \\
.17(260)\end{array}$ & $\begin{array}{l}.15(263) \\
.19(266) \\
.17(260)\end{array}$ & $\begin{array}{l}.03 \\
.08 \\
.04\end{array}$ & $\begin{array}{l}112) \\
112) \\
104)\end{array}$ & $\begin{array}{l}.951 \\
.960 \\
.950\end{array}$ & & $\begin{array}{l}.96(263) \\
.90(266) \\
.94(260)\end{array}$ \\
\hline & \multicolumn{8}{|c|}{ Negative Diagonal } \\
\hline $\begin{array}{l}1 \\
2 \\
3 \\
4\end{array}$ & $\begin{array}{l}.88(372) \\
.89(418) \\
.87(418) \\
.86(418)\end{array}$ & $\begin{array}{l}.18(251) \\
.19(265) \\
.08(266) \\
.19(266)\end{array}$ & $\begin{array}{l}11(250) \\
.11(265) \\
.11(266) \\
.18(266)\end{array}$ & & $\begin{array}{l}107) \\
112) \\
112) \\
112)\end{array}$ & $\begin{array}{l}.910 \\
.971 \\
.931 \\
.941\end{array}$ & $\begin{array}{l}51) \\
65) \\
66) \\
66)\end{array}$ & $\begin{array}{l}.96(250) \\
.93(265) \\
.97(266) \\
.95(266)\end{array}$ \\
\hline \multirow{2}{*}{4} & \multicolumn{8}{|c|}{ Four Locations } \\
\hline & \multicolumn{7}{|c|}{ One Target Present* } & $\begin{array}{l}\text { Probability of a Correct } \\
\text { Whereabouts Response }\end{array}$ \\
\hline \multirow[t]{2}{*}{ SUBJECT } & No Target & $\begin{array}{l}\text { Positive } \\
\text { Diagonal }\end{array}$ & $\begin{array}{r}\text { Nega } \\
\text { Diag } \\
\end{array}$ & & $\begin{array}{l}\text { Pos } \\
\text { Dias }\end{array}$ & $\begin{array}{l}\text { tive } \\
\text { onal }\end{array}$ & $\begin{array}{l}\mathrm{Ne} \\
\mathrm{Diz}\end{array}$ & $\begin{array}{l}\text { ative } \\
\text { gonal } \\
\end{array}$ \\
\hline & \multicolumn{8}{|c|}{ Divided Attention } \\
\hline 1 & $.810(509)$ & $.160(341)$ & .240 & & .870 & 341) & .80 & (340) \\
\hline 2 & $.750(528)$ & $.225(357)$ & $.140(3$ & & .870 & 357) & .87 & (357) \\
\hline 3 & $.770(533)$ & $.250(359)$ & .270 & & .880 & 359) & .84 & (360) \\
\hline \multirow[t]{2}{*}{4} & $.760(534)$ & $.241(360)$ & .2600 & & .860 & 360) & .85 & $(360)$ \\
\hline & \multicolumn{6}{|c|}{$\begin{array}{l}\text { Focused Attention } \\
\text { (Negative Diagonal) }\end{array}$} & & \\
\hline 1 & $.890(480)$ & $.185(319)$ & .570( & & .850 & 319) & .840 & (319) \\
\hline 2 & $.870(475)$ & $.150(318)$ & .875( & & .7800 & 318) & .885 & (318) \\
\hline 3 & $.830(480)$ & $.300(319)$ & .5600 & & .7500 & 319) & .890 & (316) \\
\hline \multirow[t]{2}{*}{4} & $.850(470)$ & $.165(310)$ & .880( & & .8011 & $310)$ & .82 & (309) \\
\hline & \multicolumn{6}{|c|}{$\begin{array}{l}\text { Focused Attention } \\
\text { (Positive Diagona!) }\end{array}$} & & \\
\hline 1 & $.870(480)$ & $.500(320)$ & .235( & & .835 & 320) & .83 & (318) \\
\hline 2 & $.850(452)$ & $.870(318)$ & .170 & & .905 & $318)$ & .82 & (318) \\
\hline 3 & $.880(480)$ & $.705(319)$ & .2351 & & .890 & 319) & & (320) \\
\hline 4 & $.865(470)$ & $.875(309)$ & .1650 & & .860 & 309) & & (310) \\
\hline
\end{tabular}

*There are few data for cases in which two targets were present, so we have not included these figures.

\section{Display-Size Effects}

In Figure 3, the display-size effect in this experiment is graphed as the probability of a correct whereabouts response for the two-location condition (averaged over positive and negative diagonal data) versus this probability for the four-location (two-diagonal), divided-attention condition. Each subject's estimated $P_{2}$ is based on about 1,000 observations, and each $P_{4}$ is based on about 720 observations. These estimates were obtained by pooling data over occurrence of the target in the different possible positions. For each subject, the data are consistent with the two attention models and are well below the boundary function. The theoretical functions in Figure 3 differ from those found in Figure 1 because, in the four-location condition in the present experiment, subjects must choose one of two diagonals rather than one of four locations. For this reason, the boundary curve was computed using a result from Green and Weber (1980) based on Luce's choice theory. The reader should consult Yellot (1977) for a discussion of the 


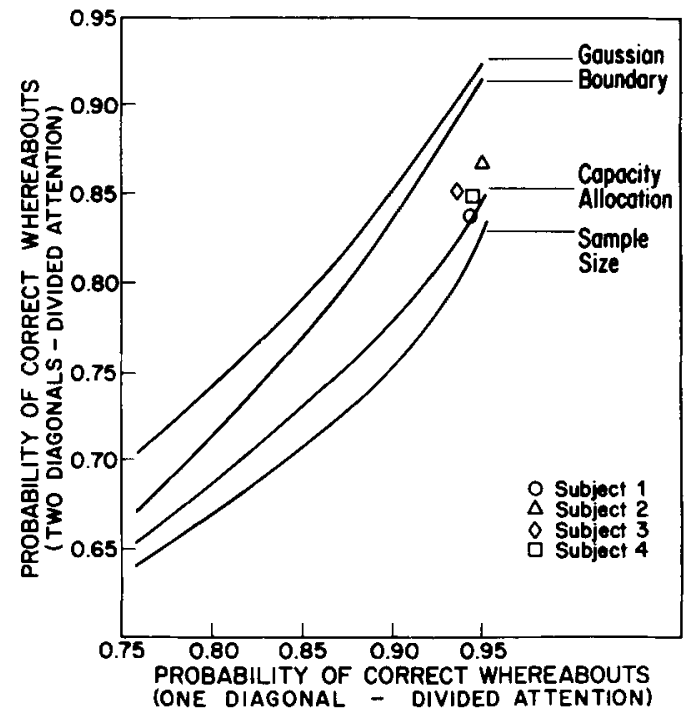

Figure 3. The relation between $P_{2}$ (probability of a correct location judgment in the two-location task) and $P_{4}$ (same probability in the four-Jocation task) predicted by the boundary curve and two attention models when the choice is between diagonals in the four-location tagk. The data points are from four subjects in the present experiment.

relation between choice theory and the distribution of the maximum of $n$ independent and identically distributed random variables. The equation is

$$
P_{4}=1-\frac{2}{3}\left[1-\frac{P_{2}}{P_{2}+3\left(1-P_{2}\right)}\right],
$$

where $\mathrm{P}_{4}$ is the probability of a correct whereabouts response given four locations and $P_{2}$ is the probability of a correct whereabouts response given two locations.

\section{Probability of a Correct Whereabouts Response}

In Figure 4, the probability of a correct whereabouts response for the two-diagonal divided-attention condition is plotted versus this probability for the focused-attention condition. Recall that the class of continuous-state models predicts that $P(C W \mid D A)$ $\geqslant P(C W \mid F A)$; this means that points in the graph should lie on or below and to the right of the diagonal. The diagonal line represents the predictions for the continuous-state model given the choice of the optimal criteria. On the other hand, the class of twostate models predicts that $P(C W \mid D A)<P(C W \mid F A)$; this means data points should lie above and to the left of the diagonal line. From Figure 4, it appears that the prediction of the class of continuous-state models is favored over the two-state model predictions by this analysis of the data.

With regard to the specific attention models being considered, the sample-size model is clearly favored over the capacity-allocation model. One additional point should be made about the prediction of the capacity-allocation model represented, under the optimal decision rule, by the dashed line in Figure 4 . This prediction is based on the assumption that the conditional probability of a correct detect state is the same as for a correct nondetect state, and that these probabilities are given by the cumulative exponential function: $1-\mathrm{e}^{-\phi}$. If the probability of a nondetect state given a distractor is smaller than the probability of a detect state given a target, then this dashed line will move closer to the diagonal. In fact, if the chance of a nondetect state, given a distractor, is very small (high false-alarm rate), then the dashed line will fall well below the diagonal. This point is discussed in Appendix 1 under two-state models. The "yes-no" data presented in Table 2 are consistent with the assumption that the probability of a nondetect state given a distractor $[q(\phi)]$ is greater than or equal to the probability of a detect state given a target $[b(\phi)]$. Because we used a procedure in which each letter could take on the role of target on one trial and distractor on another, this assumption seems to be satisfied. Thus, the dashed line in Figure 4 is representative of the capacity-allocation model prediction for these data.

\section{Focused Versus Divided Attention}

The validity of conclusions reached from the data in Figure 4 depends upon whether subjects indeed focus attention on a single diagonal and ignore the information in the unattended diagonal. We now consider, for each class of models, the consequences of a failure of subjects to focus attention when in-

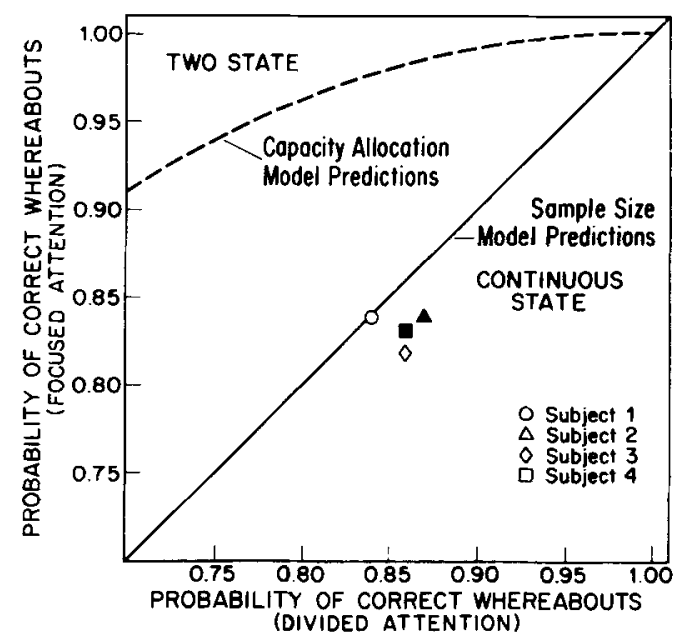

Figure 4. The relation between $\operatorname{Pr}(C W \mid D A)$ and $\operatorname{Pr}(C W \mid$ FA) for the sample-size model and the capacity-allocation model. In general, the class of continuous-state models predicts data on or below the diagonal line, while the class of two-state models predicts data above the diagonal line. The data points are from the four subjects in the present experiment. Filled symbols indicate subjects that met criteria for focused attention. 
structed to do so. Failure to focus attention may result in dividing attention either equally or unequally between diagonals and then using the same decision strategy as in the divided-attention condition. For the class of continuous-state models, this will result in points that will lie on or below the diagonal (on the diagonal if attention is divided equally and the optimal criteria are used). The same implication holds for the class of two-state models. Thus, failure of subjects to follow this instruction will probably result in data below the diagonal and consistent with the predictions of the class of continuous-state models.

The question of whether subjects did, in fact, focus attention on a single diagonal was tested using the predictions expressed in Equations 3a-3c. These predictions do not necessarily hold for the class of continuous-state models, because subjects may shift criteria from condition to condition. This fact does not affect the generality of our conclusions, however, since only the prediction of the class of two-state models is altered by a failure to focus attention. Recall that it was predicted that the probability of a "no" response given only distractors in a diagonal should be the same whether that diagonal is the only one present or simply the attended diagonal. This should be true independently of whether a target is present or absent in the unattended diagonal (Equa-

Table 3

Three Tests of Whether Subjects Ignored the Unattended Diagonal

\begin{tabular}{|c|c|c|c|}
\hline & One Diagonal & $\begin{array}{c}\text { Two Diagonals } \\
\text { (Focused Attention) }\end{array}$ & \\
\hline \multicolumn{4}{|c|}{$\begin{array}{c}\text { TEST 1 } \\
\text { (Equation 3a) }\end{array}$} \\
\hline SUBJECT & $P\left(N O \mid S_{00}\right)$ & $\begin{array}{c}P\left(N O \mid S_{1000}, S_{0100}, S_{0010}, S_{0001}\right. \\
\text { where target is in the } \\
\text { unattended diagonal) }\end{array}$ & $\mathbf{Z}$ \\
\hline $\begin{array}{l}1 \\
2 \\
3 \\
4\end{array}$ & $\begin{array}{l}.890(764) \\
.870(837) \\
.880(835) \\
.855(828)\end{array}$ & $\begin{array}{l}.535(639) \\
.872(636) \\
.630(635) \\
.877(618)\end{array}$ & $\begin{array}{r}14.89 \\
-.11 \\
11.32 \\
-.92\end{array}$ \\
\hline \multicolumn{4}{|c|}{$\begin{array}{c}\text { TEST } 2 \\
\text { (Equation 3b) }\end{array}$} \\
\hline & $P\left(N O \mid S_{00}\right)$ & $P\left(N O \mid S_{0000}\right)$ & $z$ \\
\hline $\begin{array}{l}1 \\
2 \\
3 \\
4\end{array}$ & $\begin{array}{l}.890(764) \\
.870(837) \\
.880(835) \\
.855(828)\end{array}$ & $\begin{array}{l}.880(960) \\
.870(927) \\
.850(960) \\
.860(940)\end{array}$ & $\begin{array}{r}.64 \\
.02 \\
1.86 \\
-\quad .27\end{array}$ \\
\hline \multicolumn{4}{|c|}{$\begin{array}{c}\text { TEST } 3 \\
\text { (Equation 3c) }\end{array}$} \\
\hline & $P\left(N O \mid S_{10}, S_{01}\right)$ & $\begin{array}{c}P\left(N O \mid S_{1000} S_{0100}, S_{0010}, S_{0001}\right. \\
\text { where target is in } \\
\text { the attended diagonal) }\end{array}$ & $\mathbf{z}$ \\
\hline 1 & $.125(1009)$ & $.270(637)$ & .7 .49 \\
\hline 2 & $.175(1057)$ & $.160(636)$ & .78 \\
\hline 3 & $.123(1064)$ & $.268(639)$ & -7.56 \\
\hline 4. & $.178(1052)$ & $.165(620)$ & .69 \\
\hline
\end{tabular}

tions $3 a$ and $3 b$; Tests 1 and 2 in Table 3). Also, the probability of a "no" response, given one target present, should be the same whether that diagonal is the only one present or simply the attended diagonal (Equation 3c; Test 3). The relevant detection data and the results of these tests are presented in Table 3. For these tests, the estimated probabilities were arrived at by pooling over positive and negative diagonal data. Inspection of the table reveals that only the data of Subjects 2 and 4 strongly favor the conclusion that subjects do, indeed, ignore information in the unattended diagonal. This means that we can make an inference about the two model classes for these two subjects only.

\section{DISCUSSION}

There are two types of results to be summarized from this paper. The first is the theoretical result developed in Appendix 1. There it is shown that predictions can be derived which discriminate between models of attention that assume that the internalstrength representation of stimulus information is two-valued and models that assume that the strength representation is continuous-valued. These predictions involve a comparison of target-localization performance when attention is focused on a subset of the stimulus display versus performance when attention is divided among all display locations. Specifically, the two-state model predicts that whereabouts performance with focused attention must be greater than that with divided attention. In contrast, the continuous-state model predicts that performance in the divided-attention condition will equal or exceed that in the focused-attention condition.

The second type of result derives from the empirical test of the above predictions. Data from all four subjects were consistent with the prediction of the class of continuous-state models (see Figure 4). A further analysis of the data revealed that two of the subjects were not completely successful in following focused-attention instructions (a critical assumption of our predictions), thus weakening our inferences from the whereabouts data of these subjects. Based on data of the two remaining subjects, however, we conclude that the continuous-state model provides the best account of the data.

Our predictions discriminating between two-state and continuous-state attention models may be applied to information-processing situations other than visual search. For example, consider detection and location of an auditory signal, detection and identification of pitches, and detection and identification of spatial frequencies. However, for these predictions to apply, the situation must involve a limitation on processing resources and control over the allocation of this resource. In our experiments, resource allo- 
cation was accomplished by instructing subjects to focus or divide attention. Alternatively, the experimenter may overtly control the subject's allocation of attention. For example, Shaw and Bates (Note 1) tested subjects in a visual search experiment in which only two fixations were allowed in searching for a target. When subjects were asked to optimize a whereabouts response, they allocated all their fixations to one area and guessed the ignored area when a target was not detected. In the present experiments, a similar overt control of attentional allocation could have been achieved by not displaying the ignored diagonal but still allowing it as a whereabouts choice. Other constraints on the application of our model predictions are: (1) Stimuli must be classifiable into one of two groups (e.g., target and nontarget); (2) it must be possible to experimentally approximate equal detectability of each stimulus type for all sources of information; and (3) it must be possible to put multiple sources of information into two groups and increase the division of attention by increasing the number of subcomponents in each group. In our experiment, this last constraint was satisfied by defining the areas to be attended as the diagonals of a square. This had another advantage over the use of only two positions in space. In focusing attention on a diagonal, there is no advantage to fixating the upper versus the lower position, as would be the case if subjects were instructed to focus attention on the top row versus the bottom row of our displays.

Two other aspects of the data are worthy of mention because they replicate earlier findings. The first concerns the display-size effect observed in Figure 3. This result reinforces the conclusion reached in previous experiments using similar stimuli (Shaw, 1983), that, as the number of display locations (independent sources of information) is increased from two to four, the noisiness of information obtained from each location increases. The other important result is presented in Appendix 2. Here, an analysis of the two-location detection data revealed that three of four subjects used an independent-decisions, attention-sharing rule in which it is assumed that attention is shared between stimulus locations on each trial. In previous experiments (Shaw, 1982), data from seven subjects fit either this or an alternative version of the independent-decisions model in which the amount of attention allocated to each location varies from trial to trial (the all-or-none attention model).

The investigation of detection with multiple sources of information, as discussed, for example, in Appendix 2 , is a problem for which our present test of twostate versus continuous-state attention models has important implications. It has been shown (Shaw, 1982) that an independent-decisions sharing model which assumes a two-state representation of stimulus strength (two-state, first-order integration model) makes the same predictions for "yes-no" detection data as an independent-decisions sharing model assuming a continuous-valued representation (continuous-state, second-order integration model). These two models therefore give an equally good account of much of the detection data presented both in the earlier paper and in Appendix 2 of the present paper. Our current result in favor of a continuousvalued strength representation gives us a basis for rejecting the class of two-state detection models in favor of the continuous-state models, at least for tasks and stimuli similar to ours.

In classical two-state models, sensory states above threshold can be treated as continuous; the real difference between two-state and continuous-state models, then, is whether a subject's threshold or criterion is fixed or can be varied. Previously, investigators tested the sensitivity of sensory mechanisms in "threshold" experiments in which the stimulus (target) was present on nearly all trials. A few "catch trials" (target not present) were inserted to insure that subjects did not say "yes" just because they knew that the target was always present. Relatively few catch trials were used, because it was believed that a subject's tendency to report the perception of the target was generally influenced only by whether sensory strength was above threshold, and not by possible biases in response tendencies. Classical threshold-type detection models are based on the assumption that, when a barely detectable stimulus is presented, the internal stimulus representation is in one of the two possible states, detect or nondetect. Usually, it is assumed that sensory states above threshold may have a continuous representation.

The two-state model we present is a generalization of Luce's (1963) model from the single informationsource detection situation to situations in which attention is divided among two or more sources of information, and there is a limitation on the total resource available for processing these sources. It should be noted, also, that our interpretation of the two-state model is broader than the traditional one. We assume that the model applies whenever an initial stimulus code involves categorization of a stimulus into one of two possible states for the purpose of making a decision. Our particular tests of the model do not address the question of whether this is the only code, but only whether the threshold is fixed.

In 1955, Swets, Tanner, and Birdsall questioned the assumption of a threshold that is independent of the subject's judgmental standards, and introduced the theory of signal detection as a model of the human detection process. Within this model, the internal representation of stimulus strength is conceptualized as a continuous random variable. The continuous-state model we present generalizes the 
signal-detection model to the case in which there are multiple sources of information to attend to and there is a limit on the total resources available for processing. Traditionally, it has been thought that whether an internal code involves a fixed threshold or criterion (two-state), or a criterion that can be varied (continuous-state), is not under subject control, that is, not a consequence of a subject-selected strategy. If this is the case, then the present evidence favoring continuous-state models is strong because it demonstrates the existence of a difference in performance that would result from a continuous-state model, and not a two-state model (the data from two of four subjects). However, if the correct assumption is that the choice of fixed versus variable thresholds can vary from subject to subject, or from task to task within a subject, then our results provide a way of separating these two possibilities. Only further exploration of the type we have tried can lead to a resolution of this question.

\section{REFERENCE NOTE}

1. Shaw, M. L., \& Bates, M. On the difference between detecting and locating targets. Paper presented at the meeting of the Mathematical Psychology Society, Providence, Rhode Island, August 1979.

\section{REFERENCES}

Eniksen, C. W., \& Spencer, T. Rate of information processing in visual perception: Some results and methodological considerations. Journal of Experimental Psychology Monograph, 1969, 79(2, Part 2).

Estes, W. K., \& TAylor, H. A. A detection method and probabilistic models for assessing information processing from brief visual displays. Proceedings of the National Academy of Sciences, 1964, 52, 446-454.

Gardner, G. T. Evidence for independent parallel channels in tachistoscopic perception. Cognitive Psychology, 1973, 4, 130-155.

Graham, N. Visual detection of aperiodic spatial stimuli by probability summation among narrowband channels. Vision Research, 1977, 17, 637-652.

Green, D. M., \& Birdsall, T. G. Detection and recognition. Psychological Review, 1978, 85, 192-206.

Green, D. M., \& LuCE, R. D. Speed-accuracy tradeoff in auditory detection. In S. Kornblum (Ed.), Attention and performance IV. New York: Academic Press, 1973.

Grenn, D. M., \& Swets, J. A. Signal detection theory and psychophysics. New York: Wiley, 1966.

Green, D. M., \& WeBen, D. L. Detection of temporally uncertain signals. Journal of the Acoustical Society of America, 1980, 67, 1304-1311.

Judy, P. F., Swensson, R. G., \& Massimiliano, S. Lesion detection and signal-to-noise ratio in CT images. Medical Physics, 1981, 8, 13-23.

Kadane, J. B. Optimal whereabouts search. Operations Research, 1971, 19, 894-904.

KinchlA, R. A. Temporal and channel uncertainty in detection: A multiple observation analysis. Perception \& Psychophysics, 1969, 5, 129-136.

Kinchla, R. A., \& Collyer, C. E. Detecting a target letter in briefly presented arrays: A confidence rating analysis in terms of a weighted additive effects model. Perception \& Psychophysics, 1974, 16, 117-122.
Kinchla, R. A. Detecting target elements in multi-element arrays: A confusability model. Perception \& Psychophysics, 1974, 15, 149-159.

Koopman, B. O. The theory of search: Pt. III: The optimum distribution of search effort. Operations Research, 1957, 5, 613-626.

Krantz, D. H. Threshold theories of signal detection. Psychological Review, 1969, 76, 308-324.

LUCE, R. D. A threshold model for simple detection experiments. Psychological Review, 1963, 42, 461-489.

LucE, R. D. Thurstone's discriminal processes fifty years later. Psychometrika, 1977, 42, 461-489.

LucE, R. D. Detection thresholds: A problem reconsidered. Science, 1960, 132, 1495.

MELA, D. F. Information theory and search theory as special cases of decision theory. Operations Research, 1961, 9, 907-909.

Mulligan, R., \& Shaw, M. L. Multimodal signal detection: Independent decisions vs. integration. Perception \& Psychophysics, 1980, 28, 471-478.

Mulligan, R., \& Shaw, M. L. Attending to simple auditory and visual signals. Perception \& Psychophysics, 1981, 30, 447-454.

Rollman, G. B., \& Nachmias, J. Simultaneous detection and recognition of chromatic flashes. Perception \& Psychophysics, 1972, 12, 309-314.

SACHs, M. B., Nachmias, J., \& Robson, J. G. Spatial frequency channels in human vision. Journal of the Optical Society of America, 1971, 61, 1176-1186.

Schneider, W., \& Shifrrin, R. M. Controlled and automatic human information processing: I. Detection, search and attention. Psychological Review, 1977, 84, 1-66.

Shaw, M. L. Reaction time in reading a tachistoscopic display for a memory set item. Perception \& Psychophysics, 1977, $21,15-25$.

SHAw, M. L. Identifying attentional and decision-making components in information processing. In R. S. Nickerson (Ed.) Attention and performance VIII. Hillsdale, N.J: Erlbaum, 1980.

Shaw, M. L. Attending to multiple sources of information: (l) The integration of information in decision-making. Cognitive Psychology, 1982, 14, 353-409.

SнAw, M. L. Division of attention among spatial locations: A fundamental difference between detection of letters and detection of luminance increments. In H. Bouma \& D. G. Bonwhuis (Eds.), Attention and performance $X$. Hillsdale, N.J: Erlbaum, 1983.

Shaw, M. L., \& Shaw, P. Optimal allocation of cognitive resources to spatial locations. Journal of Experimental Psychology: Human Perception and Performance, 1977, 3, 201-211.

Shiffrin, R. M., \& Gardner, G. T. Visual processing capacity and attentional control. Journal of Experimental Psychology, $1972,93,72-83$.

Starr, S. J., Metz, C. E., Lusted, L. B., \& Goodenovoh, D. J. Visual detection and localization of radiographic images. Radiology, 1975, 116, 533-538.

Stone, L. D. Theory of optimal search. New York: Academic Press, 1975.

Tognetri, K. P. An optimal strategy for a whereabouts search. Operations Research, 1968, 16, 209-211.

Wickelgren, $\mathbf{W}$. A. Strength theories of disjunctive visual detection. Perception \& Psychophysics, 1967, 2, 331-337.

YELLOT, J. I. The relationship between Luce's choice axiom, Thurstone's theory of comparative judgment and the double exponential distribution. Journal of Mathematical Psychology, $1977,15,109-144$.

\section{NOTES}

1. The relationship plotted in Figure 2 can be arrived at in the following way. First, select an arbitrary value of $\Phi$ : $P$ (correct detection $\mid \Phi=1-e^{-\Phi}$. Next, compute the same probability, 
given $\Phi / 2$ : $P\left(\right.$ correct detection $\mid \Phi / 2=1-e^{-\Phi / 2}$. Then choose a pair of correct-rejection rates, $q(\Phi)$ and $q(\Phi / 2)$, and use them to compute the difference in false-alarm rates between the dividedand focused-attention cases, $[1-q(\Phi / 2)]-[1-q(\Phi)]$, where $\mathrm{q}(\Phi)>\mathrm{q}(\Phi / 2)$. Next, calculate $\mathrm{P}(\mathrm{CW} \mid \mathrm{DA})$ and $\mathrm{P}(\mathrm{CW} \mid \mathrm{FA})$ by summing, for each case, the probability of a hit and the estimate of the probability of a correct guess given failure to detect a target. The last two steps are then repeated for different values of $q(\Phi)$ and $q(\Phi / 2)$, and the results are plotted as in Figure 2.

2. For example, $\mathbf{P}_{2}$, the probability correct in the two-location task, can be lowered or raised by decreasing or increasing display luminance. This, of course, will cause corresponding changes in $\mathrm{P}_{4}$. A change in luminance level is assumed, in the model, to change the value of the parameter $\sigma_{k}^{2}$, but not of the parameter $\Phi$, the total attention available. Thus, changing the luminance level of our stimuli is interpreted as analogous, in a real-world search problem, to altering visibility (e.g., the effect of fog) and not the total search effort. Changing the total duration of a display, however, may be viewed as changing $\Phi$, the total processing capacity (Shaw, 1977), and perhaps also changing $\sigma_{k}^{2}$. For purposes of the present analysis, it does not matter whether a given level of performance is achieved by altering display duration or luminance level.

\section{APPENDIX 1}

Two-State Model

\section{Notation}

\begin{tabular}{|c|c|}
\hline & $\begin{array}{l}\text { Location } k \text {. } \\
\text { Internal representation (code) of the stimulus in } \\
L_{k} \text { where } i=0 \text { for nontarget and } i=1 \text { for } \\
\text { target. }\end{array}$ \\
\hline & $\begin{array}{l}\text { Stimulus pattern: } i, j=0,1 \text {, depending on } \\
\text { whether nontarget or target in } L_{k} \text {. }\end{array}$ \\
\hline$\left(\mathbf{L}_{k}\right)$ & $\begin{array}{l}\text { Probability the target appears in } \mathrm{L}_{\mathrm{k}} \text {. } \\
\text { The total amount of attention, capacity, pro- } \\
\text { cessing resources, or search effort. }\end{array}$ \\
\hline$(\varphi$ & $\begin{array}{l}\text { The amount of attention allocated to } L_{k} \text {. } \\
P\left(X_{k 1}=1 ; \phi_{k}\right) \text {, the probability of detect state, } \\
D_{k} \text {, given a target in } L_{k} \text { and } \phi_{k} \text { is allocated } \\
\text { there. }\end{array}$ \\
\hline$\left(\phi_{1}\right)$ & $\begin{array}{l}P\left(X_{k 0}=0 ; \phi_{k}\right) \text { the probability of a nondetect } \\
\text { state, } \bar{D}_{k} \text {, given a nontarget in } L_{k} \text { and } \phi_{k} \text { is } \\
\text { allocated there. }\end{array}$ \\
\hline $\begin{array}{l}\mathbf{R}_{\mathbf{i}} \\
\mathrm{FA}^{\prime}\end{array}$ & $\begin{array}{l}\text { Correct whereabouts response. } \\
\text { Correct detection response. } \\
\text { Choice of } \mathrm{L}_{\mathrm{i}} \text { in whereabouts task. } \\
\text { Focused attention. } \\
\text { Divided attention. }\end{array}$ \\
\hline
\end{tabular}

\section{Representation Assumptions}

(1) $\Phi \geqslant 0 \quad$ Total capacity is nonnegative.

(2) $\Phi=\Sigma \phi_{\mathbf{k}} \quad$ Capacity is additive or conserved.

(3) $b\left(\phi_{k}\right) \quad$ Right continuous, concave, and increasing.

(4) $q\left(\phi_{k}\right) \quad$ Right continuous, concave, and increasing.

In our application of this model, we have chosen an empirical situation for which it makes sense to assume that $b\left(\phi_{k}\right)=q\left(\phi_{k}\right)$ for all $\phi_{k}$. In other applications, it might make sense to assume that $X_{k i}$ always starts out with the value 0 and with some probability changes to the value 1 . In other applications, there might be reason to assume that $q\left(\phi_{k}\right) \geqslant b\left(\phi_{k}\right)$ or that $b\left(\phi_{k}\right)>q\left(\phi_{k}\right)$ for all $\phi_{k}$. It is also possible that the relation between $b\left(\phi_{k}\right)$ and $q\left(\phi_{k}\right)$ changes with Q.

\section{Probability of a Correct Whereabouts Search}

Here we treat only the case of two locations. In our experiment, these are the positive and negative diagonals.

Focused attention. This is also called the whereabouts strategy: attend to one diagonal, and if no target is detected, guess the unattended diagonal. It follows that

$$
\operatorname{Pr}(C W \mid F A)=\operatorname{Pr}\left(L_{k}\right) q(\Phi)+\operatorname{Pr}\left(L_{j}\right) b(\Phi),
$$

where $L_{j}$ is the attended diagonal and $L_{k}$ is the ignored diagonal. For convenience, we assume $\operatorname{Pr}\left(L_{1}\right) \geqslant \operatorname{Pr}\left(L_{2}\right)$. The two possible whereabouts strategies are obvious and, for each, the probability of a correct whereabouts response is given by:

(1) attend to $L_{2}$,

$$
\operatorname{Pr}(C W \mid F A)=\operatorname{Pr}\left(L_{1}\right) q(\Phi)+\operatorname{Pr}\left(L_{2}\right) b(\Phi)
$$

(2) attend to $\mathrm{L}_{1}$,

$$
\operatorname{Pr}(C W \mid F A)=\operatorname{Pr}\left(L_{1}\right) b(\Phi)+\operatorname{Pr}\left(L_{2}\right) q(\Phi)
$$

What is the better strategy? If either $\operatorname{Pr}\left(L_{1}\right)=\operatorname{Pr}\left(L_{2}\right)$ or $q(\Phi)=b(\Phi)$, it does not matter and, as we show later, the whereabouts (FA) strategy gives a higher $\operatorname{Pr}(C W)$ than the divided-attention strategy. If we assume $\operatorname{Pr}\left(L_{1}\right) \geqslant \operatorname{Pr}\left(L_{2}\right)$, then which location should be ignored depends on the relation between $q(\Phi)$ and $b(\Phi)$.

Divided attention. Here the strategy is to divide attention among the locations in order to maximize the probability of a correct detection response and then choose the location most likely to contain the target (i.e., the location having highest posteriori probability for the target, given the pattern of detect states and the amount of attention allocated to each location). Choosing the location with the greatest posterior probability is the best strategy given that one must divide attention (i.e., cannot use the whereabouts strategy) and at the same time try to maximize $\operatorname{Pr}(C W \mid D A)$; therefore, it is sufficient to show that the best $\operatorname{Pr}(C W \mid D A)$ is always smaller than the best $\operatorname{Pr}(\mathrm{CW} \mid \mathrm{FA})$.

The probability of a correct whereabouts response given divided attention is

$$
\operatorname{Pr}(C W \mid D A)=\operatorname{Pr}\left(R_{1} \mid L_{1}\right) \operatorname{Pr}\left(L_{1}\right)+\operatorname{Pr}\left(R_{2} \mid L_{2}\right) \operatorname{Pr}\left(L_{2}\right)
$$

Now, $\operatorname{Pr}\left(R_{1} \mid L_{1}\right)$ must be decomposed into four components:

$$
\begin{aligned}
& \operatorname{Pr}\left(R_{1} \mid X_{11}=1, X_{20}=0\right) \operatorname{Pr}\left(X_{11}=1, X_{20}=0 \mid L_{1}\right), \\
& \operatorname{Pr}\left(R_{1} \mid X_{11}=1, X_{20}=1\right) \operatorname{Pr}\left(X_{11}=1, X_{20}=1 \mid L_{1}\right), \\
& \operatorname{Pr}\left(R_{1} \mid X_{11}=0, X_{20}=1\right) \operatorname{Pr}\left(X_{11}=0, X_{20}=1 \mid L_{1}\right), \\
& \operatorname{Pr}\left(R_{1} \mid X_{11}=0, X_{20}=0\right) \operatorname{Pr}\left(X_{11}=0, X_{20}=0 \mid L_{1}\right) .
\end{aligned}
$$


The same applies, of course, to $\operatorname{Pr}\left(R_{2} \mid L_{2}\right)$. For the sake of simplifying notation, we shorten $\left(X_{k i}=0\right)$ to $\bar{D}_{k}$ and $\left(X_{k i}=1\right)$ to $D_{k}$.

Given a pattern of detect and nondetect states over locations, the objective is to specify for that pattern which diagonal should be given as the whereabouts response so that the overall probability of a correct whereabouts response is maximized. The overall probability of a correct whereabouts response will then be a mixture of strategies that depends upon these patterns of detect and nondetect states. Thus, we first consider these patterns of detect states and the corresponding optimal whereabouts response. In all the cases below, it is assumed that the optimal allocation function $(\Phi)$ for detection is used and there is sufficient $\Phi$ for this plan to result in some positive allocation to each location. If there is not sufficient $\Phi$, then the best choice of a location, given failure to detect in the high-probability location, $L_{1}$, is still the high-probability location, $L_{1}$. This follows because under the optimal detection plan, $\Phi$ is allocated entirely to $L_{1}$, and this means

$$
\operatorname{Pr}\left(L_{1}\right)[1-b(\Phi)]>\operatorname{Pr}\left(L_{2}\right) \text {. }
$$

In other words, the conditional posterior probability for $L_{1}$ is still higher than the prior probability for $\mathrm{L}_{2}$. (See Stone, 1975.)

We now consider, one by one, the optimal choice for each pattern of detect states.

Case 1: $\mathrm{D}_{1} \overline{\mathrm{D}}_{2}\left(\mathrm{X}_{1 \mathrm{i}}=1\right.$ and $\left.\mathrm{X}_{2 \mathrm{j}}=0\right)$.

(a) $\operatorname{Pr}\left(L_{1} \mid D_{1} \bar{D}_{2}\right)=\frac{b\left(\phi_{1}\right) q\left(\phi_{2}\right) \operatorname{Pr}\left(L_{1}\right)}{\operatorname{Pr}\left(D_{1} \bar{D}_{2}\right)}$

(b) $\operatorname{Pr}\left(L_{2} \mid D_{1} \bar{D}_{2}\right)=\frac{\left[1-q\left(\phi_{1}\right)\right]\left[1-b\left(\phi_{2}\right)\right] \operatorname{Pr}\left(L_{2}\right)}{\operatorname{Pr}\left(D_{1} \bar{D}_{2}\right)}$

The optimal strategy is to choose $L_{1}$ if $\operatorname{Pr}\left(L_{1} \mid D_{1} \bar{D}_{2}\right)>$ $\operatorname{Pr}\left(L_{2} \mid D_{1} \bar{D}_{2}\right)$; otherwise, choose $L_{2}$. We consider the case in which $\operatorname{Pr}\left(L_{1}\right)=\operatorname{Pr}\left(L_{2}\right)$. The optimal allocation of $\Phi$ is $\phi_{1}=$ $\phi_{2}$, so we have the following decision rule: Choose $L_{1}$ if

$$
b(\Phi / 2) q(\Phi / 2) \geqslant[1-b(\Phi / 2)][1-q(\Phi / 2)],
$$

or when

$$
b(\Phi / 2) \geqslant 1-q(\Phi / 2) .
$$

If $b(\phi)=q(\phi)$, then the decision rule reduces to choosing $L_{1}$ if

$$
b(\Phi / 2) \geqslant 1 / 2 \text {. }
$$

Case 2: $\bar{D}_{1} D_{2}\left(X_{1 i}=0\right.$ and $\left.X_{2 j}=1\right)$.

The analysis of this case gives the same result as Case 1, when $L_{2}$ is substituted for $L_{1}$.

Case 3: $D_{1} D_{2}\left(X_{1 i}=1\right.$ and $\left.X_{2 j}=1\right)$.

(a) $\operatorname{Pr}\left(L_{1} \mid D_{1} D_{2}\right)=\frac{b\left(\phi_{1}\right)\left[1-q\left(\phi_{2}\right)\right] \operatorname{Pr}\left(L_{1}\right)}{\operatorname{Pr}\left(D_{1} D_{2}\right)}$ (b) $\operatorname{Pr}\left(L_{2} \mid D_{2} D_{2}\right)=\frac{\left[1-q\left(\phi_{1}\right)\right]\left[b\left(\phi_{2}\right)\right] \operatorname{Pr}\left(L_{2}\right)}{\operatorname{Pr}\left(D_{1} D_{2}\right)}$

The optimal strategy is to choose $L_{1}$ if

$$
\left[b\left(\phi_{1}\right)\right]\left[1-q\left(\phi_{2}\right)\right] \operatorname{Pr}\left(L_{1}\right) \geqslant\left[1-q\left(\phi_{1}\right)\right]\left[b\left(\phi_{2}\right)\right] \operatorname{Pr}\left(L_{2}\right) .
$$

We first consider the case in which $\operatorname{Pr}\left(\mathrm{L}_{1}\right)=\operatorname{Pr}\left(\mathrm{L}_{2}\right)$ so that $\phi_{1}=\phi_{2}$. The optimal choice of a location is $L_{1}$ if

$$
\mathrm{b}(\Phi / 2)[1-\mathrm{q}(\Phi / 2)] \geqslant[1-\mathrm{q}(\Phi / 2)] \mathrm{b}(\Phi / 2) .
$$

But the left and right sides are equal, so the choice is arbitrary.

Case 4: $\overline{\mathrm{D}}_{1} \overline{\mathrm{D}}_{2}\left(\mathrm{X}_{1 \mathrm{i}}=0\right.$ and $\left.\mathrm{X}_{2 \mathrm{j}}=0\right)$.

(a) $\operatorname{Pr}\left(\mathrm{L}_{1} \mid \overline{\mathrm{D}}_{1} \overline{\mathrm{D}}_{2}\right)=\frac{\left[1-\mathrm{b}\left(\phi_{1}\right)\right] \mathrm{q}\left(\phi_{2}\right) \operatorname{Pr}\left(\mathrm{L}_{1}\right)}{\operatorname{Pr}\left(\overline{\mathrm{D}}_{1} \overline{\mathrm{D}}_{2}\right)}$

(b) $\operatorname{Pr}\left(\mathrm{L}_{2} \mid \overline{\mathrm{D}}_{1} \overline{\mathrm{D}}_{2}\right)=\frac{\left[\mathrm{q}\left(\phi_{1}\right)\right]\left[1-\mathrm{b}\left(\phi_{2}\right)\right] \operatorname{Pr}\left(\mathrm{L}_{2}\right)}{\operatorname{Pr}\left(\overline{\mathrm{D}}_{1} \overline{\mathrm{D}}_{2}\right)}$

The optimal strategy is to choose $\mathrm{L}_{\mathbf{2}}$ if

$$
P\left(L_{2}\right) q\left(\phi_{1}\right)\left[1-b\left(\phi_{2}\right)\right]>\left[1-b\left(\phi_{1}\right)\right] q\left(\phi_{2}\right) P\left(L_{1}\right) .
$$

Here we consider the case in which $P\left(L_{1}\right)=P\left(L_{2}\right)$ and so $\phi_{1}=\phi_{2}$. The optimal choice is $L_{2}$ if

$$
\mathrm{q}(\Phi / 2)[1-\mathrm{b}(\Phi / 2)] \geqslant[1-\mathrm{b}(\Phi / 2)] \mathrm{q}(\Phi / 2) .
$$

But these are equal, so the choice is arbitrary.

We now derive an expression for $\operatorname{Pr}(C W \mid D A)$ when $\operatorname{Pr}\left(L_{1}\right)=\operatorname{Pr}\left(L_{2}\right)$ and the optimal response selection rule is used for the case in which $b(\Phi)>1-q(\Phi)$ : The conditional probability of a detect state, given the target is present, is at least as great as when the target is not present.

$$
\begin{aligned}
\operatorname{Pr}(C W \mid D A)= & \operatorname{Pr}\left(R_{1} \mid L_{1}\right) \operatorname{Pr}\left(L_{1}\right)+\operatorname{Pr}\left(R_{2} \mid L_{2}\right) \operatorname{Pr}\left(L_{2}\right) \\
= & \operatorname{Pr}\left(D_{1} \mid L_{1}\right) \operatorname{P}\left(\bar{D}_{2} \mid L_{1}\right) \operatorname{Pr}\left(L_{1}\right) \\
& +\operatorname{Pr}\left(D_{1} \mid L_{1}\right) \operatorname{Pr}\left(D_{2} \mid L_{1}\right) \operatorname{Pr}\left(L_{1}\right)(1 / 2) \\
& +\operatorname{Pr}\left(\bar{D}_{1} \mid L_{1}\right) \operatorname{Pr}\left(\bar{D}_{2} \mid L_{1}\right) \operatorname{Pr}\left(L_{1}\right)(1 / 2) \\
& +\operatorname{Pr}\left(\bar{D}_{1} \mid L_{2}\right) \operatorname{Pr}\left(D_{2} \mid L_{2}\right) \operatorname{Pr}\left(L_{2}\right) \\
& +\operatorname{Pr}\left(\bar{D}_{1} \mid L_{2}\right) \operatorname{Pr}\left(\bar{D}_{2} \mid L_{2}\right) \operatorname{Pr}\left(L_{2}\right)(1 / 2) \\
& +\operatorname{Pr}\left(D_{1} \mid L_{2}\right) \operatorname{Pr}\left(D_{2} \mid L_{2}\right) \operatorname{Pr}\left(L_{2}\right)(1 / 2)
\end{aligned}
$$

Simplifying and substituting the model expressions in the equation, we have

$$
\begin{aligned}
\operatorname{Pr}(C W \mid D A)= & b\left(\phi_{1}\right) q\left(\phi_{2}\right) P\left(L_{1}\right) \\
& +b\left(\phi_{1}\right)\left[1-q\left(\phi_{2}\right)\right] P\left(L_{1}\right) \\
& +q\left(\phi_{1}\right) b\left(\phi_{2}\right) \operatorname{Pr}\left(L_{1}\right)
\end{aligned}
$$




$$
\begin{aligned}
& +\mathrm{q}\left(\phi_{1}\right) \mathrm{b}\left(\phi_{2}\right) \mathrm{P}\left(\mathrm{L}_{2}\right) \\
& +\mathrm{q}\left(\phi_{1}\right)\left[1-\mathrm{b}\left(\phi_{2}\right)\right] \mathrm{P}\left(\mathrm{L}_{2}\right) \\
= & \mathrm{b}(\Phi / 2) \operatorname{Pr}\left(\mathrm{L}_{1}\right)+\mathrm{q}(\Phi / 2) \operatorname{Pr}\left(\mathrm{L}_{2}\right) \\
= & .5[\mathrm{~b}(\Phi / 2)+\mathrm{q}(\Phi / 2)] .
\end{aligned}
$$

Comparison of $\operatorname{Pr}(\mathrm{CW} \mid \mathrm{DA})$ and $\operatorname{Pr}(\mathrm{CW} \mid \mathrm{FA})$

and

$$
\operatorname{Pr}(\mathrm{CW} \mid \mathrm{FA})=.5[\mathrm{~b}(\Phi)+\mathrm{q}(\Phi)]
$$

$$
\operatorname{Pr}(\mathrm{CW} \mid \mathrm{DA})=.5[\mathrm{~b}(\Phi / 2)+\mathrm{q}(\Phi / 2)] .
$$

Since $b(\Phi)>b(\Phi / 2)$ and $q(\Phi / 2)$, it follows that

or

$$
.5[\mathrm{~b}(\Phi)+\mathrm{q}(\Phi)]>.5[\mathrm{~b}(\Phi / 2)+\mathrm{q}(\Phi / 2)]
$$

$$
\operatorname{Pr}(\mathrm{CW} \mid \mathrm{FA})>\operatorname{Pr}(\mathrm{CW} \mid \mathrm{DA}) \text {. }
$$

The reverse prediction obtains, however, if $b(\phi)<$ $1-q(\phi)$, that is, if conditional probability of a detect state, given the target is present, is smaller than the probability of this state when the target is not present. We leave this proof to the reader.

\section{Continuous-State Model}

\section{Additional Notation}

$f_{t}(X), f_{d}(X)$ The probability density function for target and distractor random variables, respectively.

$\mu_{2}, \mu_{d} \quad$ The mean of the target and distractor random variable, respectively.

\section{Representation Assumptions}

(1) $\Phi \geqslant 0$

(2) $\Phi=\Sigma \phi_{k}$

(3) $\tilde{\sigma}_{k}^{2}=\frac{\sigma_{k}^{2}}{\phi_{k}}$

(4) $f_{d}(X)=f_{t}(X+C)$

(5) $f_{d}, f_{t}$
Total capacity is nonnegative.

Capacity is additive (conserved under partitions of it).

Variance of $\mathbf{X}_{\mathrm{ki}}$ is inversely proportional to $\phi_{k} ; \sigma_{k}^{2}$ is the variance inherent in the stimulus representation.

The probability distribution of $\mathbf{X}_{\mathbf{k} 1}$ is a shift of the probability distribution of $X_{\mathbf{k} 0}$.

Symmetric, unimodal distributions with finite means and variances.

For convenience, we assume that $\mu_{\mathrm{d}}=0$. The assumptions that $f_{d}$ and $f_{t}$ are symmetric and unimodal with the same variance inherent in the stimulus representation are analogous to the assumption that $b\left(\phi_{k}\right)=q\left(\phi_{k}\right)$ in the two-state model.

\section{Probability of a Correct Whereabouts Search: \\ Two Locations}

Focused attention (whereabouts strategy). The subject is instructed to attend to one diagonal, and if the target is not detected, to guess the unattended diagonal. For the continuous-state model, we assume that the subject picks a criterion $\beta_{k}$ for the attended location $L_{k}$, and if $X_{k i}<\beta_{k}$, then the unattended location is selected for the whereabouts response. Thus, if $\mathrm{L}_{\mathrm{k}}$ is the attended location:

$$
\begin{aligned}
\operatorname{Pr}(C W \mid F A)= & \operatorname{Pr}\left(X_{k 0}<\beta_{k}\right)\left[1-\operatorname{Pr}\left(L_{k}\right)\right] \\
& +\operatorname{Pr}\left(X_{k 1}>\beta_{k}\right) \operatorname{Pr}\left(L_{k}\right) .
\end{aligned}
$$

We denote by $G(Z)$ the standardized cumulative distribution function and then write

$$
\begin{aligned}
\operatorname{Pr}(C W \mid F A)= & G\left[\frac{\beta_{k}-\mu_{\mathrm{e}}}{\sigma / \sqrt{\Phi}}\right]\left[1-P\left(L_{k}\right)\right] \\
& +\left[1-G\left(\frac{\beta_{k}-\mu_{4}}{\sigma / \sqrt{\Phi}}\right)\right] P\left(L_{k}\right)
\end{aligned}
$$

If it is assumed that $\operatorname{Pr}\left(\mathrm{L}_{k}\right)=1-\operatorname{Pr}\left(\mathrm{L}_{\mathrm{k}}\right)$ and $\beta_{k}$ is optimal $\left[\beta_{k}=\left(\mu_{t} / 2\right)\right.$ will maximize the probability of a correct detection at the attended location], then it follows that

$$
\operatorname{Pr}(\mathrm{CW} \mid \mathrm{FA})=\mathrm{G}\left[\begin{array}{c}
+\sqrt{\Phi} \mu_{\underline{L}} \\
2 \boldsymbol{2} \sigma
\end{array}\right]
$$

Divided attention (detection strategy). The subject is instructed to attend equally to both locations (diagonals) and choose one location (diagonal) most likely to contain the target given the impressions from the brief flash. In the model, we assume that the subject chooses the location with the largest random variable. Thus,

$$
\begin{aligned}
\operatorname{Pr}(C W \mid D A)= & \operatorname{Pr}\left(L_{1}\right) \operatorname{Pr}\left(X_{20}-X_{11}<0\right) \\
& +\operatorname{Pr}\left(L_{2}\right) \operatorname{Pr}\left(X_{10}-X_{21}<0\right) .
\end{aligned}
$$

Again, letting $G$ denote the standardized distribution function, the equation above can be written as follows:

$$
\operatorname{Pr}(C W \mid D A)=\operatorname{Pr}\left(L_{1}\right) G\left[\frac{0-\mu_{t}}{\sqrt{2 \sigma^{2} / \Phi}}\right]+\operatorname{Pr}\left(L_{2}\right) G\left[\frac{0-\mu_{t}}{\sqrt{2 \sigma^{2} / \Phi}}\right]
$$

Because $\operatorname{Pr}\left(L_{1}\right)=\operatorname{Pr}\left(L_{2}\right)$, we have

$$
\operatorname{Pr}(C W \mid D A)=G\left[\frac{+\sqrt{\Phi} \mu_{\mathrm{t}}}{2 \sigma}\right]
$$

Comparing the final form for $\mathrm{P}(\mathrm{CW} \mid \mathrm{DA})$ and the final form for $P(C W \mid F A)$, we see $\operatorname{Pr}(C W \mid D A)=\operatorname{Pr}(C W \mid F A)$. If subjects use a nonoptimal $\beta$ in the focused attention condition, we will have $\operatorname{Pr}(C W \mid F A)<\operatorname{Pr}(C W \mid D A)$.

\section{Probability of a Correct Whereabouts Search: \\ n Locations}

In the case of $\mathrm{n}$ locations, we have Monte Carlo results for the Gaussian probability function and the assumption that $\operatorname{Pr}\left(L_{k}\right)=\operatorname{Pr}\left(L_{j}\right)$ for all $k$ and $j$.

Whereabouts strategy. Here one location, $L_{k}$, is chosen to be ignored; if, for all $s \neq k, X_{s i}<\beta_{s}$, then $L_{k}$ is chosen for the whereabouts responses. If, however, there are some 
Table A1

Continuous-State Model: Examples from Monte Cario Study

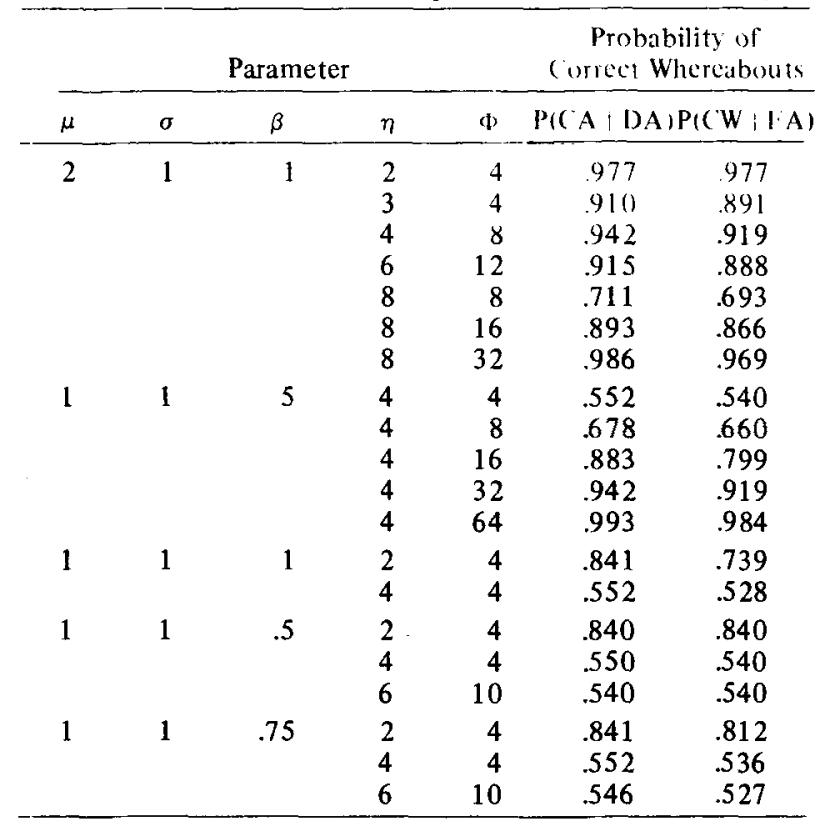

$X_{\mathrm{si}}$ such that $\mathrm{X}_{\mathrm{si}}>\beta_{\mathrm{s}}$, then $\mathrm{L}_{\mathrm{r}}$ is chosen such that $L_{\mathrm{r}}=$ $\max \left\{X_{s i}\right\}$. The probability of a correct choice under this strategy is

$$
\begin{aligned}
\operatorname{Pr}(C W \mid F A)= & \operatorname{Pr}\left(L_{k}\right) \prod_{s \neq k} G\left(\frac{\beta_{s}}{\sigma / \sqrt{\phi_{i}}}\right) \\
& +\sum_{s \neq k} \operatorname{Pr}\left(L_{s}\right) \int_{\beta_{k}}^{\infty} g\left(\frac{X-\mu_{t}}{\sigma / \sqrt{\phi_{s}}}\right) \prod_{k, i \neq s} G\left(\frac{x}{\sigma / \sqrt{\phi_{i}}}\right) d x .
\end{aligned}
$$

Detection strategy. Again, it is assumed that all locations are equally likely to contain the target. The lecation chosen $\mathrm{L}_{\mathrm{k}}$, for the whereabouts response is assumed to be the one such that $\mathrm{X}_{\mathrm{ki}}=\max \left\{\mathrm{X}_{\mathrm{j}}\right\}$. Unlike the whereabouts strategy, attention may be allocated to all locations so that the probability of a correct detection response is minimized. Since $\operatorname{Pr}\left(L_{k}\right)=\operatorname{Pr}\left(L_{i}\right)$ for all $k, i$, this leads to $\phi_{i}=\phi_{k}$ for all $\mathrm{i}, \mathrm{k}$. We now write the probability of a correct whereabouts response as a function of this detection strategy (DA) as

$$
\operatorname{Pr}(C W \mid D A)=\int_{-\infty}^{\infty} g\left(\begin{array}{l}
X-\mu_{s} \\
\sigma / \frac{\mid \Phi}{n}
\end{array}\left(\frac{X}{\sigma} / \frac{\Phi}{n}\right)^{n-1} \mathrm{dx}\right.
$$

In Table A1, we present some sample computations of $\mathrm{P}(\mathrm{CW} \mid \mathrm{FA})$ and $\mathrm{P}(\mathrm{CW} \mid \mathrm{DA})$ for various values of $\mu, \sigma, \beta$, $\Phi$, and $n$ (number of locations). These computations show that dividing attention equally among the cells and then choosing the maximum random variable provides a higher probability of a correct whereabouts response than the whereabouts strategy in which a location is ignored.

\section{APPENDIX 2}

In a recent paper, Shaw (1982) discussed a wide range of decision models for detection with multiple sources of information. Models were broadly categorized along two dimensions: (1) the nature of the initial stimulus strength representation (e.g., two-state, three-state, integer-state, continuous-valued) and (2) the extent of processing that takes place before strength measures from different stimulus sources are combined to form the integrated code on which a "yes-no" decision is subsequently based. Two classes of decision models were differentiated on this second dimension: first-order integration models and secondorder integration models.

Models of the first-order integration type postulate an initial strength measure of each stimulus source $X_{\mathrm{ki}}$; this variable may be either discrete or continuous. These initial measures are then integrated to form a decision variable, $y$, a weighted linear combination of these values:

$$
y=w_{1} X_{1 i}+w_{2} X_{2 i}+\cdots w_{n} X_{n i} .
$$

If the decision variable y exceeds a criterion value, $\beta$, the observer says "yes"; otherwise the observer says "no." For the case when the variable $X_{k i}$ is two-valued, this model has been called a discrete-sum model (Green \& Birdsall, 1978). If the variable $X_{k i}$ is assumed to be continuous and to have a Gaussian probability function, this model is termed Gaussian integration. The class of twostate models considered earlier in this paper is very similar to the discrete-sum model.

Second-order integration models assume two levels of code before the evaluation of the integrated code, $y$. Thus, for each initial code, $\mathbf{X}_{\mathrm{k}}$, a decision variable, $\mathbf{X}_{\mathbf{k}}^{\prime}$, is constructed as follows:

$$
\begin{aligned}
& X_{k i}^{\prime}=0 \text { if } X_{k j}<\beta_{k}, \\
& X_{k i}^{\prime}=1 \text { if } X_{k i} \geqslant \beta_{k} .
\end{aligned}
$$

The observer says "yes," target present, if $y=\Sigma_{k} X_{k i}^{\prime}>c$, that is, when $c$ or more decision variables $\left(X_{k j}^{\prime}\right)$ indicate target present. Second-order integration models have also been called independent-decisions models (Mulligan \& Shaw, 1980; Shaw, 1980), probability summation models (Graham, 1977; Sachs, Nachmias, \& Robson, 1971), extreme detector models (Judy, Swensson, \& Massimiliano, 1981; Starr, Metz, Lusted, \& Goodenough, 1975), and independent threshold detectors (Green \& Birdsall, 1978).

For both first-order and second-order integration models, Shaw (1982) developed separate predictions for twostate, three-state, integer-counting, and continuous-state models of the initial stimulus-strength measure. Two experiments were completed using stimuli and procedures essentially identical to those in the two-location condition of the present experiment. Data from the seven subjects in these experiments were tested against predictions of the various models and were best fit by one of two versions ("fixed sharing" or "all-or-none") of the continuous-state, second-order integration model. However, comparable versions of the two-state, first-order model make precisely the same predictions for "yes-no" data as the continuousstate, second-order models and, therefore, fit the data equally well. All other models tested (three-state, integercounting, and continuous-state first-order models) were rejected.

One of the purposes of including the detection task in the present experiment was to replicate these earlier results. 
Table A2

Standard Normal Scores for Tests of the Fixed-Sharing Model

$$
\text { Two Locations }
$$

\begin{tabular}{ccc} 
Subject & Positive Diagonal & Negative Diagonal \\
\hline 1 & .18 & -.48 \\
2 & -.11 & 1.62 \\
3 & -3.17 & -2.56 \\
4 & -.31 & -.51
\end{tabular}

With this aim, the "yes-no" data from the two-location conditions (see Table 2) were compared with the predictions of several of the models discussed in Shaw (1982). Once again, the data were best fit by the common predictions of the two-state, first-order and continuous-state, second-order models. Due to significant differences in performance on the two diagonals for some subjects, the data for each diagonal were analyzed separately. Results of the test for the continuous-state, second-order fixed sharing model are given in Table A2. The test for this model is based on the prediction that

$$
P_{i j}=P\left(X_{1 i}<\beta_{1}\right) P\left(X_{2 i}<\beta_{2}\right)
$$

After logarithmic transformation, it can be shown that

$$
\ln P_{11}+\ln P_{00}=\ln P_{10}+\ln P_{01}
$$

The following statistic, derived in Shaw (1980), is then used to test for deviations from the additive relationship in Equation A2:

$$
Z=\frac{\ln P_{11}+\ln P_{00}-\ln P_{10}-\ln P_{01}}{S},
$$

where $S$ is the standard deviation of the statistic in the numerator.

Values in Table A2 greater than 1.96 indicate significant deviation from additivity and, thus, evidence against the model. The fixed-sharing models (two-state, first-order and continuous-state, second-order) were supported in six of the eight cases tested. As in the earlier experiments, all of the other models were rejected.

(Manuscript received August 19, 1981; revision accepted for publication December 6,1982 .) 